\title{
Public awareness of malignant melanoma risk factors in Germany
}

\author{
Annette Pfahlberg, Olaf Gefeller, Klaus F Kölmel
}

\begin{abstract}
Study objective-To evaluate the effects of a German public education campaign which aimed to improve knowledge on risk factors for malignant melanoma.

Design-Comparison of data from two successive cross sectional surveys conducted before (spring 1993) and after (autumn 1994) the campaign.

Setting-All 56 nursery schools in Göttingen, the capital of southern Lower Saxony, Germany.

Participants-Parents of children attending the nursery schools. Altogether 1341 questionnaires from parents were included in the first survey (response rate $64.9 \%$ ) and 1150 questionnaires in the second survey (response rate $61.4 \%$ ).

Main results-The respondents in the second survey were much better at distinguishing true melanoma risk factors from false ones. The distribution of scores measuring the degree of accurate knowledge about melanoma risks indicated that this had improved significantly $(p<0.001)$. The most pronounced change with regard to knowledge of single risk factors could be observed for "sunburn during childhood," which was correctly identified by $63.1 \%$ in the first survey and by $85.6 \%$ in the second. Substantial improvement in accurate knowledge about the influence of constitutional skin factors-number of naevi, skin type etc-was also found.

Conclusions-Notwithstanding the methodological problems in this analysis (nonrandomised design, only before and after comparison, no control group, number of non-respondents), it is concluded that this campaign improved understanding of the risks of melanoma. Continuing public education activities should be implemented to sustain and improve further knowledge on prevention of malignant melanoma.
\end{abstract}

$(\mathcal{F}$ Epidemiol Community Health 1997;51:698-700)

Malignant melanoma is increasing rapidly in incidence in fair skinned populations around the world. ${ }^{1}$ Numerous epidemiological studies in the past two decades have contributed to a better understanding of the aetiology of the disease (for overviews see ${ }^{2-4}$, for a bibliography of all 117 case-control studies on malignant melanoma published up to the end of 1995 see ${ }^{5}$ ). There is now some agreement that constitutional factors (such as skin type, number of naevi, hair colour ${ }^{367}$ ) and exposure to ultraviolet light (especially intermittent excessive sunlight exposure and sunburn during childhood $^{8-10}$ ) play an important part in the aetiology of melanoma.

Public health campaigns which aim to educate the general population about the risk factors for malignant melanoma have been launched in several countries. ${ }^{11-13}$ Because of the epidemiological evidence concerning the importance of melanoma prevention during childhood, these activities were not only addressed to the general population but were specifically targetted at the parents of young children. In Germany, such a campaign was launched in the summer of 1993: it was called "child and sun". ${ }^{14}$

There is debate over whether or not these campaigns really improve preventive knowledge, attitudes and behaviour in the population. We describe the results of two cross sectional surveys in Göttingen which were conducted before and after an intensive, nationwide public education campaign in Germany. The aim was to evaluate the effects of these activities on the knowledge about risk factors for malignant melanoma of parents of children in nursery schools.

\section{Methods}

The target population comprised parents with children aged 3-6 years living in Göttingen. We approached all 56 nursery schools and distributed (with the help of the nursery school teachers) a standardised questionnaire to all parents via their children. This procedure was implemented first in spring 1993 and repeated, using an identical procedure, in autumn 1994. In the meantime, starting before the German summer holidays of 1993, an intensive education campaign devoted to the hazards of unprotected exposure to sunlight during childhood took place nationwide in Germany. In both cross sectional surveys, the questionnaire contained a list of nine exposures and parents had to judge whether or not these would act as a melanoma risk factor. In addition to true risk factors, this list included exposures that have attracted public awareness, but have not been identified as risk factors for melanoma (such as presence of allergies, rising air pollution, unhealthy diet). From the individual answers a "knowledge score" was created and this was classified into three broad categories ("low", "medium", and "high").

Results

Although the parents were approached via their children in this study, the family 
Table 1 Detailed overview of sample sizes and response calculations in two cross sectional surveys in Göttingen

\begin{tabular}{lll}
\hline Components of the response calculation & $\begin{array}{l}\text { 1st survey } \\
\text { Spring 1993 }\end{array}$ & $\begin{array}{l}\text { 2nd survey } \\
\text { Autumn 1994 }\end{array}$ \\
\hline $\begin{array}{l}\text { A: distributed questionnaires } \\
\text { B: total number of children the 56 nursery schools are in }\end{array}$ & 2536 & 2302 \\
$\quad$ charge oft & 2306 & 2092 \\
C: estimated "double counts" and "no show" $¥$ & 241 & 219 \\
D: returned questionnaires & 1341 & 1150 \\
E: "net" response rate (\%)\} $&{64.9} &{61.4} \\
{\hline}$
\end{tabular}

*Due to typical short term fluctuations in the population covered by the nursery school, a $10 \%$ "reserve" was added to $B$.

†Based on telephone information by the nursery school 1 month before the survey.

$\ddagger$ Number of children who are living in households in which another child also attends a nursery school ("double counts") or who were absent during the entire period of the survey ("no shows"); estimation is based on the information of a subsample of 12 nursery schools in the second survey. CCalculated as D / (B-C).

Table 2 Proportions of study subjects judging different exposures as risk factors for melanoma in two cross sectional surveys in Göttingen

\begin{tabular}{llll}
\hline & $\begin{array}{l}\text { 1st survey } \\
\text { Spring 1993 } \\
(n=1341) \%\end{array}$ & $\begin{array}{l}\text { 2nd survey } \\
\text { Autumn 1994 } \\
(n=1150) \%\end{array}$ & $\begin{array}{l}\text { Statistical } \\
\text { evaluation of } \\
\text { differences } \\
p \text { value }\end{array}$ \\
\hline Exposure & 63.1 & 85.6 & $<0.001$ \\
Sunburn during childhood & 70.4 & 66.9 & 0.07 \\
Chronic sun exposure (eg farmers) & 63.5 & 62.2 & 0.50 \\
Sunburn during life & 47.4 & 56.8 & $<0.001$ \\
High number of naevi & 44.5 & 53.5 & $<0.001$ \\
Fair skin, fair hair & 45.2 & 42.7 & 0.22 \\
Intermittent intensive sun exposure (eg during & & & \\
$\quad$ holidays) & 50.3 & 39.9 & $<0.001$ \\
Rising air pollution & 16.5 & 15.7 & 0.62 \\
Presence of allergies & 13.3 & 13.0 & 0.86 \\
Unhealthy diet & & & \\
\hline
\end{tabular}

${ }^{\star} \mathrm{p}$ values derived from Fisher's exact test.

Table 3 Comparison of the score distributions measuring preventive knowledge on melanoma risk factors between two cross sectional surveys in Göttingen

\begin{tabular}{llcl}
\hline Knowledge score & $\begin{array}{l}\text { 1st survey } \\
\text { Spring 1993 } \\
(n=1341)\end{array}$ & $\begin{array}{l}\text { 2nd survey } \\
\text { Autumn 1994 } \\
(n=1150)\end{array}$ & $\begin{array}{l}\text { Statistical evaluation } \\
\text { of differences } \\
\text { p value }\end{array}$ \\
\hline Mean (SD) & $4.9(4.7)$ & $6.4(4.5)$ & $<0.001 \dagger$ \\
Categories (\%): & 20.2 & 31.0 & $<0.001 \ddagger$ \\
High & 63.8 & 59.0 & \\
Medium & 15.9 & 9.9 & \\
Low & & &
\end{tabular}

* Score distributions in both surveys are rather symmetric. Nevertheless, standard deviations (SD) are hard to interpret for arbitrarily valued scores but have been reported to indicate that variability of the score is stable over the two surveys.

tp value derived from Wilcoxon-Mann-Whitney test.

$\neq \mathrm{p}$ value derived from $\chi^{2}$ test.
KEY POINTS

- Two cross sectional surveys were conducted among parents of nursery school children-one before and one after a German public education campaign on risk factors for malignant melanoma.

- Respondents in the second survey were much better at distinguishing between true and false risk factors.

- The most pronounced change was in the number who identified sunburn in childhood as an important risk factor-this had been a particular focus of the education campaign.

- Despite some methodological problems, it is concluded that the campaign improved understanding of the risk factors for malignant melanoma.

increases the risk of developing melanoma?" The most dramatic shift-from $63.1 \%$ to $85.6 \%$ - was observed for the item "sunburn during childhood". This reflects the fact that the German campaign focused particularly on this issue. In addition, a substantial improvement in knowledge about the influence of physiological skin factors was evident $(47.4 \%$ to $56.8 \%$ for the importance of high numbers of naevi and $44.5 \%$ to $53.5 \%$ for fair skin/hair). Furthermore, the unjustified belief that increasing air pollution contributes to the occurrence of melanoma was mentioned much less often in the second survey (from $50.3 \%$ to $39.9 \%$ ).

Comparison of the distribution of the scores in the surveys (see table 3 ) emphasises the fact that respondents in 1994 were much more able to distinguish true melanoma risk factors from false ones. A significant shift of the whole score distribution towards a better level of knowledge and, when considering the categorised score, a pronounced increase in numbers in the "high knowledge" category (from $20.2 \%$ to $31.0 \%$ ) was observed in the second survey.

household was the natural sampling unit of the investigation. Detailed information on the sample sizes and response calculations are given in table 1 . The response rate was above $60 \%$ for both parts of the study. Most of the reduction in the absolute number of parents who responded in 1994 resulted from a decrease of children in the target population because of demographic changes in Germany. Altogether 2491 questionnaires from family households were included in this analysis. In the first survey, 134 of the 1341 questionnaires were completed during a personal interview with one parent. As we observed no discrepancies in results between the two types of information assessment we combined these data for all analyses, and in the second survey obtained information only by written questionnaires. Most of the questionnaires were filled out by the mothers.

Table 2 compares the results of the two cross sectional surveys concerning the proportions of parents who answered "yes" to the question "Do you think that the corresponding exposure

\section{Discussion}

Based on large samples of participants, our study provides evidence that knowledge of the risk factors for malignant melanoma is increasing among parents of young children. This has followed intensive educational efforts devoted to the prevention of malignant melanoma in Germany. The design of our investigationthat of two successive cross sectional surveyshas a major limitation with regard to its ability to prove the efficacy of the public education campaign. Since we have neither a (randomly selected) control group nor an individual follow up of study participants, changes in the response patterns cannot directly be attributed to the campaign. ${ }^{15} 16$

The observed improvement may also reflect temporal changes in other areas relevant for health education (eg, information about the topic through the media, the physicians, health insurance companies, etc). Such an effect cannot be ruled out unless the development of knowledge is studied simultaneously in a 
control group. This was impossible to implement in our situation as the campaign took place on a nationwide level. Even if some of the supporting activities in the media and elsewhere are triggered by the campaign, their beneficial effect cannot be completely attributed to the campaign. There are strong indications that influences besides the "child and sun" activities played a part in disseminating accurate information on melanoma risk factors. Media coverage of the topic increased substantially during this period, and other aspects such as the more informative advertising of sunscreens or changes in the style of reporting in fashion and beauty magazines (similar to the earlier trends in Australia ${ }^{17}$ ) may have contributed to the positive findings of our study. A strong argument in favour of the conclusion that at least a substantial part of the improvement is due to the campaign can, however, be drawn from the observation that the most pronounced increase in accurate knowledge could be seen with respect to harmful effect of sunburn during childhood. This particular aspect constituted the main focus of the "child and sun" campaign.

The lack of individual follow up of study participants may also have distorted our results. Different selection biases might have operated during the first and second surveys and these might have resulted in a changed sample composition concerning relevant variables influencing the parents' answering behaviour. As we have no information about non-respondents we cannot analyse this issue completely. We achieved a similar response rate of above $60 \%$ in both years. In addition, the distribution of standard sociodemographic variables among responding parents (age, sex, education) was nearly identical in the two surveys. Thus, we believe that this design deficiency had no substantial impact on our results.

In this paper we investigated only changes in the knowledge about risk factors for malignant melanoma. This assessment of information was based on questionnaires which are an appropriate source for this purpose. Preventive knowledge does not necessarily translate into behavioural changes; an increased level of accurate knowledge on melanoma risks would not automatically lead to parents taking better protective measures against sunburn in their children. ${ }^{1819}$ Assessing the preventive behaviour of the parents with regard to protecting their children from the harmful effects of sun is, however, difficult. ${ }^{20}$ Data from questionnaires do often not provide a valid basis for such analyses, because the parents' answers do not correspond to their actual behaviour in many cases. ${ }^{2}$

A complete evaluation of the effects of our public education campaign should comprise analysis of the changes in knowledge, attitudes, behaviour, and the direct consequences of the behaviour (for example, the frequency of sunburns). This investigation aimed to clarify the first point, other analyses are needed to complete the picture. But even at this stage, we feel confident in concluding that the German campaign had some beneficial effects on the level of understanding of the risks of melanoma. Thus public education activities should be implemented as a continuing process to sustain and, it is hoped, further improve preventive knowledge on this issue.

The study has been partially supported by a grant of the Niedersächsische Krebsgesellschaft. The authors thank Ms U Cleffmann, Mrs B Kropik, Ms H Thölking and all nursery school teachers in Göttingen for their help during the field phase of the study. The two reviewers deserve special thanks for their valuable comments on an earlier version of this paper.

1 Swerdlow AJ. International trend in cutaneous melanoma. Ann NY Acad Science 1990;609:235-51.

2 Evans RD, Kopf AW, Lew RA, et al. Risk factors for the development of malignant melanoma: review of case control studies. $\mathcal{F}$ Derm Surg Oncol 1988;14:393-408.

3 Bliss JM, Ford D, Swerdlow AJ, et al. Risk of cutaneous melanoma associated with pigmentation characteristics and freckling: systematic overview of 10 case-control studies. Int F Cancer 1995;62:367-76.

4 Koh HK. Cutaneous melanoma. $N$ Engl f Med 1991;325: 171-82.

5 Gefeller O, Hassan K, Siefker U. Eine Bibliographie epidemiologischer Fall-Kontroll-Studien zum malignen Melanom. Göttingen: Georg-August-Universität Göttingen, 1996. Technical Report ST96/04.

6 Elwood JM, Whitehead SM, Davison J, Stewart M, Galt M. Malignant melanoma in England: risks associated with Malignant melanoma in England: risks associated with
naevi, freckles, social class, hair colour and sunburn. Int $\mathcal{F}$ naevi, freckles, social class,
Epidemiol 1990;19:801-10.

7 Garbe C, Büttner P, Weiß J, Soyer HP, Stocker U, Krüger S, et al. Risk factors for developing cutaneous melanoma and criteria for identifying persons at risk: Multicenter case-control study of the central malignant melanoma registry of the German Dermatological Society. $\mathcal{f}$ Invest Dermatol 1994;102:695-9.

8 Elwood JM. Melanoma and sun exposure: contrast between intermittent and chronic exposure. World $\mathcal{F}$ Surg 1992;16: 157-65.

9 Østerlind A, Tucker MA, Stone BJ, Jensen OM. The Danish case-control study of cutaneous malignant melanoma. II. Importance of UV-light exposure. Int 7 Cancer 1988;42. 319-24.

10 Zanetti R, Franceschi S, Rosso S, Colonna S, Bidoli E. Cutaneous melanoma and sunburns in childhood in a Southern European population. Eur f Cancer 1992; 28A:1172-6.

11 Reintgen D. Primary prevention activities for malignant melanoma in the United States. In: MacKie RM, ed.
Primary and secondary prevention of malignant melanoma Primary and secondary prevention of malignant
(pigment cell, vol 11). Basel: Karger, 1996: 43-73.

12 Hughes BR. Primary prevention activities in the United Kingdom. In: MacKie RM, ed. Primary and secondary prevention of malignant melanoma (pigment cell, vol 11). Basel: Karger, 1996: 74-92.

13 Marks R. Programmes for the primary prevention of melanoma in Australia. In: MacKie RM, ed. Primary and secondary prevention of malignant melanoma (pigment cell, vol 11). Basel: Karger, 1996: 93-110.

14 Kölmel KF, Roser M, Cleffmann U, Breitbart W. Sonne, Kind und Melanoma. Melanomprävention im Kindesalter. Der Kinderarzt 1993;24:470-81.

15 Hill D, Borland R. Methodological issues in research on primary and secondary prevention of malignant melanoma. In: MacKie RM, ed. Primary and secondary prevention of malignant 1960 : $1-21$.

16 Elwood JM, Morris JM. Evaluation of programmes to modify sun exposures. In: MacKie RM, ed. Primary and secondary prevention of malignant melanoma (pigment cell, vol 11). Basel: Karger, 1996: 111-27

17 Chapman S, Marks R, King M. Trends in tans and skin protection in Australian fashion magazines 1982 through 1991. Am F Public Health 1992;82:1677-80.

18 Bourke JF, Graham-Brown RAC. Protection of children against sunburn: a survey of parental practice in Leicester. Br f Dermatol 1995;133:264-6.

19 Bastuji-Garin S, Grognard C, Grosjean F, Grob JJ, Guillaume JC. Melanoma: evaluation of a health education campaign for primary schools. Nouv Dermatol 1995; 14(suppl 1):31.

20 Bennetts K, Borland R, Swerissen H. Sun protection behaviour of children and their parents at the beach. Psychol Health 1991;5:279-87.

21 Rostaing G. Public health campaign: photoprotection of children on the beach of the Côte d'Azur during summer 1994. Nouv Dermatol 1995;14 (suppl 1):30. 